\title{
Höhere Mortalität wegen zu geringer Aufnahmekapazität
}

\author{
Manche Patienten, die von einer Therapie auf einer Intensiv- \\ station profitieren würden, können dort nicht behandelt \\ werden, weil die Station keine Aufnahmemöglichkeiten mehr \\ hat. R. Robert et al. sind nun der Frage nachgegangen, wie sich \\ dies auf die Mortalität auswirkt. \\ Am J Respir Crit Care Med 2012; 185: 1081-1087
}

An der französischen Studie beteiligten sich 10 Intensivstationen in einer Region mit der niedrigsten Intensivbettendichte. Die Autoren unterteilten die Patienten, die auf einer Intensivstation behandelt werden sollten, in 2 Gruppen: Patienten, die sofort auf der Intensivstation aufgenommen wurden und solche, deren Aufnahme aufgrund kompletter Belegung nicht möglich war. Patienten, die nicht auf die Intensivstation aufgenommen wurden, weil sie für zu krank oder zu gesund erachtet wurden, um von einer dortigen Behandlung zu profitieren, wurden von der Analyse ausgeschlossen. Die Gruppe mit abgelehnter Aufnahme wurde nochmals in 3 Subgruppen unterteilt: Patienten, die niemals auf die Intensivstation aufgenommen wurden, solche, die unmittelbar nach Verlegung eines anderen Patienten aufgenommen wurden und solche, die bei einem nachfolgenden Versuch aufgenommen wurden.

\section{Frühe Aufnahme ist entscheidend}

$\nabla$

Die 10 teilnehmenden Intensivstationen verfügten über insgesamt 131 Betten. Während des Studienzeitraums waren

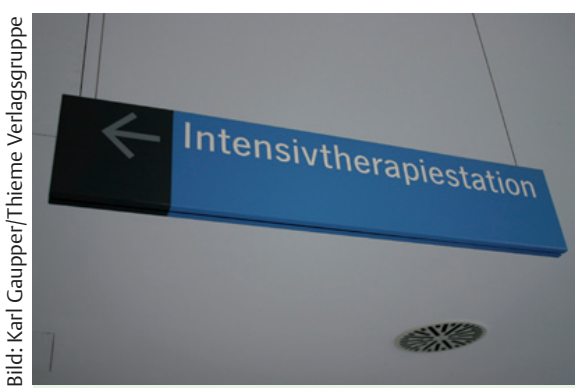

Laut französischer Studie ist eine späte Aufnahme auf der Intensivstation mit einer höheren Mortaliät assoziiert. die Stationen im Mittel an 43,6 Tagen (48,5\%) voll belegt. In die Analyse gingen 1332 Patienten ein, von denen 1139 unmittelbar auf eine Intensivstation aufgenommen und 193 wegen kompletter Belegung zunächst abgelehnt wurden. Von diesen wiederum wurden 65 niemals aufgenommen, die restlichen 128 entweder zu einem späteren Zeitpunkt ( $\mathrm{n}=89$ ) oder nach Verlegung eines anderen Patienten ( $n=39$ ). Die Zeit bis zur Aufnahme lag bei den unmittelbar aufgenommenen
Patienten im Median bei 80 min, bei den anderen Patienten bei 195 min (Verlegung eines anderen Patienten) bzw. 309 min (erneuter Versuch). Die Mortalitätsraten an den Tagen 28 und 60 betrugen in der unmittelbar aufgenommenen Gruppe 24,3 bzw. 27,2\%, bei abgelehnter Aufnahme 30,1 bzw. 33,3\% ( $p=0,07$ bzw. $\mathrm{p}=0,06)$. In der Kaplan-Meier Kurve fand sich eine deutlich höhere Mortalitätsrate bei Patienten mit initial abgelehnter Aufnahme mit 21,9 vs. $15,6 \%$ an Tag 7 . Patienten mit abgelehnter Aufnahme hatten gegenüber unmittelbar aufgenommenen an den Tagen 28 und 60 ein deutlich höheres Mortalitätsrisiko mit Odds Ratios von $1,78(\mathrm{p}=0,05)$ und 1,83 $(\mathrm{p}=0,04)$.

\section{Fazit}

Die Ergebnisse zeigen, dass eine frühe Aufnahme auf eine Intensivstation entscheidend ist und dass eine inadäquate Verfügbarkeit von Intensivbetten zu unnötigen Todesfällen führen kann, so die Autoren.

Dr. Johannes Weiß, Bad Kissingen 